\title{
Introduction: Frontiers of Electron Microscopy in Materials Science
}

The Ninth Frontiers of Electron Microscopy in Materials Science Conference (FEMMS 2003) was held October 5-10, 2003 at the Claremont Resort and Spa in Berkeley, CA. Major sponsors for this meeting included Lawrence Livermore National Laboratory, Argonne National Laboratory, Lawrence Berkeley National Laboratory, Brookhaven National Laboratory, Frederick Seitz Materials Research Laboratory, Oak Ridge National Laboratory, National Science Foundation, and University of California at Davis. Sponsors also included LEO Electron Microscopy Ltd. (Carl Zeiss SMT), E. A. Fischione, Inc., Gatan, Inc., Thermo NORAN (Thermo Electron Corp.), FEI Company, Hitachi-HHTA, JEOL USA, Inc., Seiko Instruments, and CEOS GmbH.

Session topics included analytical electron microscopy, FIB, imaging, optics and detector developments for materials science, EBSD and diffraction methods, X-ray phase contrast microscopy, electron tomography and biomaterials, nanomaterials, in-situ and time-resolved microscopies, and next generation electron microscopes and facilities. This international meeting was attended by more than 140 participants. Manuscripts addressing new techniques or the initial application of a technique were published in Ultramicroscopy (vol. 103, no. 1, April 2005), but the more applied electron microscopy application papers are being published in this issue of Microscopy and Microanalysis.

Wayne King

Lawrence Livermore National Laboratory

Guest Editor 